\title{
ANÁLISE CRÍTICA DA CAPACIDADE PREDITIVA DE DOIS MODELOS DE AVALIAÇÃ̃O DO RISCO À SAÚDE HUMANA PELA EXPOSIÇÃO AO CHUMBO EM CRIANÇAS DE 0 A 7 ANOS DE IDADE
} TO HUMAN HEALTH BY EXPOSURE TO LEAD IN CHILDREN FROM 0 TO 7 YEARS OF AGE

\section{Gustavo Alonso Muñoz Magna (iD)}

Doutor em Ciências: Energia e Ambiente pela Universidade Federal da Bahia (UFBA). Professor da Faculdade de Tecnologia e Ciências (FTC) - Salvador (BA), Brasil.

\section{Sandro Lemos Machado (D)}

Doutor em Geotecnia pela Universidade de São Paulo (USP). Professor titular da UFBA - Salvador (BA), Brasil.

\section{Miriam de Fátima Carvalho (i)}

Doutora em Geotecnia Universidade de São Paulo (USP). Professora adjunta I da UFBA - Salvador (BA), Brasil.

\section{Milton José Porsani (D)}

Doutor em Geofísica pela Universidade Federal da Bahia (UFBA). Professor titular da UFBASalvador (BA), Brasil.

\section{Endereço para correspondência:} Gustavo Alonso Muñoz Magna Avenida Luís Viana, 8.812 - Paralela -CEP: 41741-590-Salvador (BA), Brasil-E-mail: ingmag@gmail.com

Recebido em: 13/08/2019

Aceito em: 20/12/2019

\section{RESUMO}

Analisou-se a capacidade preditiva de dois modelos de avaliação do risco pela exposição ao chumbo $(\mathrm{Pb})$ em crianças de 0 a 7 anos residentes em uma área comprovadamente contaminada por metais. Os modelos utilizados foram o integrated exposure uptake biokinetic (IEUBK), criado pela Agência de Proteção Ambiental dos Estados Unidos, e o public health assessment process, desenvolvido pela Agência de Substâncias Tóxicas e Registro de Doenças (ATSDR), também dos Estados Unidos. Dois cenários de exposição foram simulados: o primeiro considerado padrão, e o segundo, individualizado. Foram utilizados antecedentes de uma base de dados gerada mediante estudos anteriores realizados na área contendo valores individuais de concentração de $\mathrm{Pb}$ no solo superficial, poeira domiciliar e alimentos vegetais cultivados em quintais e consumidos pela população local. Para verificar as estimativas de ambos os modelos, valores de $\mathrm{Pb}$ no sangue ( $\mathrm{Pb}-\mathrm{S})$, obtidos de forma experimental em crianças residentes na área de estudo, foram utilizados. Os dois modelos, após calibração, aproximaramse do valor médio de $\mathrm{Pb}-\mathrm{S}$ obtido experimentalmente $\left(4,25 \mu \mathrm{g} \cdot \mathrm{dL}^{-1}\right.$ ATSDR; 4,17 $\mu \mathrm{g} \cdot \mathrm{dL}^{-1}$ IEUBK e 4,56 $\mu \mathrm{g} \cdot \mathrm{dL}^{-1}$ experimental). Não foram detectadas diferenças estatisticamente significativas entre os modelos $(p=0,393)$ quando avaliados os valores médios estimados de $\mathrm{Pb}-\mathrm{S}$ no cenário sem consumo de vegetais. Foi possível observar associação linear entre os valores individuais de $\mathrm{Pb}$-S previstos pelo modelo da ATSDR e os calculados pelo modelo IEUBK $(r=0,60)$. Ambos os modelos se apresentaram adequados, quando ajustados, para predizer o risco pela exposição ao $\mathrm{Pb}$ em crianças, contudo o modelo IEUBK apresentou maior praticidade relacionada à economia de recursos e ao tempo para predizer o risco pela exposição ao $\mathrm{Pb}$.

Palavras-chave: contaminação; saúde ambiental; passivo ambiental; predição de riscos; metais.

\section{ABSTRACT}

The predictive capacity of two risk assessment models was analyzed by exposure to lead $(\mathrm{Pb})$ in children aged 0 to 7 years living in an area known to be contaminated with metals. The models used were the Integrated Exposure Uptake Biokinetic (IEUBK) and the Public Health Assessment Process, developed by the US Agency for Toxic Substances and Disease Registry. Two exposure scenarios were simulated, the first considered standard and the second individualized. We used antecedents from a database generated by previous studies carried out in the area containing individual values of $\mathrm{Pb}$ concentration in the superficial soil, household dust and vegetable foods grown in backyards and consumed by the local population. To verify the estimates of both models, blood $\mathrm{Pb}(\mathrm{Pb}-\mathrm{S})$ values obtained experimentally 
in children living in the study area were used. Both models, after calibration, approached an experimental mean value of Pb-S (4.25 $\mu \mathrm{g} . \mathrm{dL}^{-1}$ ATSDR; $4.17 \mu \mathrm{g} . \mathrm{dL}^{-1}$ IEUBK; $4.56 \mu \mathrm{g} . \mathrm{dL}^{-1}$ experimental). No statistically significant differences were detected between the models $(p=0.393$ ) when the estimated average Pb-S values in the scenario without vegetable consumption were evaluated. It was possible to observe a linear association between the individual $\mathrm{Pb}-\mathrm{S}$ values predicted by the ATSDR model and those calculated by the IEUBK model $(r=0.60)$. Both models were adequate, when adjusted, to predict the risk of $\mathrm{Pb}$ exposure in children; however, the IEUBK model showed greater resource and timesaving practicality to predict the risk of $\mathrm{Pb}$ exposure.

Keywords: contamination; environmental health; environmental liabilities; risk prediction, metals.

\section{INTRODUÇÃO}

A avaliação de risco à saúde humana constitui uma das melhores ferramentas disponíveis para dar suporte, com base científica, à tomada de decisões no gerenciamento do risco em uma área contaminada (SUÁREZ; ARIAS; CASTAÑEDA, 2007). A vantagem fundamental da aplicação das metodologias de avaliação de riscos é precisamente a oportunidade de incorporar os conhecimentos científicos no processo de tomada de decisões e assim estabelecer de forma clara e com maior confiança as bases da avaliação, diferenciando os critérios que condicionam a tomada de decisão final, tais como viabilidade técnica e custo econômico e social.

As diferentes metodologias de avaliação de risco existentes são similares no que diz respeito à necessidade de quantificação das variáveis populacionais e aos potenciais efeitos dos contaminantes sobre uma população-alvo de estudo por diferentes vias de exposição (CAPELETI et al., 1998), contudo o paradigma da avaliação de riscos à saúde humana não está isento de incertezas, produto principalmente da variabilidade intrínseca das populações avaliadas e dos métodos utilizados para estimação do risco. O resultado da estimação do risco sobre a saúde humana é fortemente influenciado pelas atividades desenvolvidas na etapa de avaliação da exposição. Nessa fase, utiliza-se grande quantidade de fontes de informação e técnicas, e, embora sejam realizadas mensurações diretas dos contaminantes, diversos critérios, parâmetros e inferências devem ser assumidos sobre as diferentes variáveis consideradas na avaliação. Nesse sentido, é provável que nem sempre todos os dados estejam disponíveis. Portanto, a utilização de modelos alternativos pode representar uma opção a ser considerada para a realização da avaliação, seja de forma exclusiva, seja de forma complementar.

A complexidade das interações entre os diversos fatores que influenciam a migração dos poluentes, desde seu lançamento até atingirem a população receptora, além dos diferentes critérios e das suposições utilizados nas distintas etapas da metodologia, e ainda quando usados diferentes modelos de avaliação, impõe incertezas ao resultado do real risco que representa determinado cenário de contaminação. $O$ desconhecimento das incertezas associadas aos valores estimados de risco para uma área contaminada e dos parâmetros que são necessários para seu cálculo constitui um grande problema no momento da tomada de decisão de implementação de medidas mitigadoras, para minimizar a exposição ambiental e/ou reduzir os prováveis efeitos na saúde humana da população exposta. Segundo De Miguel (2003), por conta das enormes implicações econômicas, no caso de adoção ou não de medidas mitigadoras, é indispensável contar com uma medida de fiabilidade nos resultados da avaliação do risco.

Nesse contexto, faz-se necessário analisar a capacidade preditiva dos modelos de avalição de risco comumente empregados, sendo eles tradicionais, alternativos e/ ou complementares, apontando os seus pontos fortes, suas deficiências, vantagens e desvantagens, para dessa forma auxiliar no processo de escolha do modelo a ser utilizado em determinado caso de contaminação. O objetivo deste estudo foi avaliar a capacidade preditiva de dois modelos de avaliação de risco pela exposição ao chumbo $(\mathrm{Pb})$ em crianças de 0 a 7 anos residentes em uma área contaminada. 


\section{Modelo da Agência de Substâncias Tóxicas e Registro de Doenças (Agency for Toxic Substances and Disease Registry)}

Neste caso, a avaliação de riscos à saúde humana corresponde à metodologia de caracterização de áreas contaminadas e de tomada de decisões na gestão de risco formalizada, que foi desenvolvida pela National Research Council (NRC) em 1983, aplicada pela Agência de Proteção Ambiental dos Estados Unidos (United States Environmental Protection Agency - USEPA), mediante o Programa Superfund, e adaptada pela Agência de Substâncias Tóxicas e Registro de Doenças (Agency for Toxic Substances and Desease Registry - ATSDR, 2005), especificamente para avaliar o risco à saúde humana. O objetivo da análise de riscos é proporcionar, por meio da estimação quantitativa ou qualitativa, uma ideia acerca da possibilidade da ocorrência de efeitos adversos na saúde dos seres humanos pela exposição a contaminantes presentes em um local contaminado.

Segundo De Miguel (2003), o processo tradicional de avaliação de riscos à saúde humana é composto de quatro etapas:

- identificação do perigo;

- avaliação toxicológica;

- avaliação da exposição;

- caracterização do risco.

A identificação do perigo visa ao estudo das propriedades físico-químicas e toxicológicas dos contaminantes presentes na área, características físicas do local, além de características das atividades humanas (uso do solo) e da identificação da população potencialmente exposta aos contaminantes. Os resultados mais relevantes dessa etapa são a determinação das variáveis de exposição da população e o desenvolvimento do modelo conceitual (MC) da área contaminada (DE MIGUEL, 2003).

Por outro lado, a avaliação toxicológica tem por objetivos a identificação dos elementos ou compostos potencialmente adversos à saúde humana e o estudo da relação dose-resposta. Essa etapa é caracterizada de acordo com o efeito ou com a resposta tóxica nos seres humanos, classificando-se os contaminantes de interesse em cancerígenos e não cancerígenos. Para aqueles elementos ou substâncias apontados como não cancerígenos, admite-se que existe um intervalo de magnitude de exposição que vai desde zero até um valor máximo que pode ser considerado tolerável pelo organismo, sem apresentar efeitos tóxicos significativos, o que deriva na definição de valores de referência para doses de exposição, tais como: ingestão diária tolerável (IDT), da Organização Mundial da Saúde (OMS); doses de referência (RfD), da USEPA; e doses de mínimo risco (MRL), da ATSDR.

A terceira etapa, a avaliação da exposição, estima o tipo e a magnitude da exposição dos receptores aos contaminantes de interesse presentes ou migrando da área contaminada. A magnitude da exposição expressa-se na determinação das doses diárias de exposição ao(s) contaminante(s) pela população. Essa etapa é realizada para cada rota de exposição definida na etapa de identificação do perigo.

A etapa final da metodologia corresponde à caracterização do risco, que consiste na definição qualitativa e quantitativa, com base na integração das informações proporcionadas pelas etapas de avaliação toxicológica e avaliação da exposição. Basicamente essa etapa se expressa na comparação entre os níveis de exposição e os efeitos estimados para o(s) contaminante(s). Ou seja, estima-se o nível de risco mediante a comparação das doses de exposição dos indivíduos com as doses que originam efeitos adversos à saúde humana.

\section{Modelo biocinético e de exposição integrado (integrated exposure uptake biokinetic)}

O modelo biocinético e de exposição integrado (integrated exposure uptake biokinetic - IEUBK) é utilizado na avaliação do risco pela exposição ao $\mathrm{Pb}$ ambiental exclusivamente em crianças de 0 a 7 anos de idade, por meio da predição da concentração de chumbo no sangue ( $\mathrm{Pb}-\mathrm{S}$ ) e da probabilidade de detectar valores acima de níveis considerados como de referência de exposição e, consequentemente, de risco (USEPA, 2007). Segundo Caballero et al. (2017), o modelo IEUBK é indicado para ser utilizado em estudos ambientais sobre contaminação por $\mathrm{Pb}$ quando avaliações do metal no sangue 
são menos viáveis de serem desenvolvidas. Os principais parâmetros de entrada correspondem a valores de concentração de $\mathrm{Pb}$ em diferentes compartimentos ambientais, tais como ar, dieta alimentar, solo, poeira domiciliar, água, vegetais e leite materno, além de valores de biodisponibilidade dos diversos compartimentos avaliados e informações sobre hábitos de crianças. Esses valores são tidos como predefinidos no modelo, podendo ser modificados em função da disponibilidade de dados e adequação ao cenário de exposição.

Na predição dos níveis de $\mathrm{Pb}-\mathrm{S}$ como consequência da exposição ambiental ao $\mathrm{Pb}$, o modelo utiliza quatro componentes:

- exposição;

- absorção;

- biocinético;

- variabilidade (USEPA, 2007).

\section{Biomarcador de exposição}

A utilização de biomarcadores de exposição objetiva diminuir as incertezas na estimação do risco, associada ao uso dos métodos matemáticos tradicionais de avaliação. Pode-se dizer que, embora o uso de biomarcadores torne economicamente mais oneroso o processo de avaliação do risco, os resultados e as conclusões justificam plenamente o seu custo (DÍAZ-BARRIGA; ORELLANA, 1999).

O biomarcador de exposição estima a dose interna por meio da determinação do elemento químico ou de seu produto biotransformado em fluidos biológicos, tais como sangue, urina, ar exalado e outros, possibilitando a quantificação da substância no organismo, quando a toxicocinética é bem conhecida (AMORIM, 2003). Em termos de acurácia e precisão analítica, o sangue é um dos biomarcadores mais vantajosos ao se tratar do $\mathrm{Pb}$. O uso da medida do $\mathrm{Pb}-\mathrm{S}$ é amplamente difundido e recorrente como teste laboratorial para aferir o grau de exposição ao metal.

A medida desse metal no sangue pode refletir a exposição recente ao $\mathrm{Pb}$ do meio externo, assim como o $\mathrm{Pb}$ mobilizado do compartimento ósseo (SCHWARTZ; HU, 2007). Comumente, a maior parte do $\mathrm{Pb}-\mathrm{S}$ reflete a exposição recente (semanas e meses), uma vez que o $\mathrm{Pb}$ tem um tempo de permanência relativamente curto no
O componente da exposição está relacionado aos níveis do contaminante detectados nos compartimentos ambientais sob análise e às suas taxas de ingestão diária $\left(\mu \mathrm{g} . \mathrm{dia}^{-1}\right)$ pelas crianças, tendo como resultado a dose total de ingestão diária de $\mathrm{Pb}$. Já o componente de absorção utiliza os valores fornecidos pelo componente de exposição para calcular a proporção da dose diária total ingerida do metal que é transferida ao plasma sanguíneo e a proporção que será eliminada do organismo. Por outro lado, o componente biocinético calcula a quantidade de $\mathrm{Pb}$ em cada compartimento-alvo do organismo (plasma, rins, tecidos moles, entre outros) em função da idade, como resultado dos processos fisiológicos e bioquímicos. Taxas de transferência entre os compartimentos e mecanismos de eliminação são estimadas para obter os níveis de $\mathrm{Pb}-\mathrm{S}$ de crianças entre 0 e 7 anos de idade. Por último, o componente de variabilidade utiliza a média geométrica do nível de $\mathrm{Pb}-\mathrm{S}$ de acordo com os parâmetros estimados pelos componentes anteriores do modelo, para calcular a distribuição de probabilidade log-normal dos níveis de $\mathrm{Pb}-\mathrm{S}$.

sangue, cerca de um mês (BARBOSA JR. et al., 2005). Entretanto, há situações em que a maior parte do $\mathrm{Pb}-\mathrm{S}$ pode não refletir a exposição ao metal presente no meio externo, mas sim o $\mathrm{Pb}$ depositado nos ossos, como é o caso de situações caracterizadas por altas taxas de remodelamento ósseo, como o crescimento, a gravidez e o período pós-menopausa (SCHWARTZ; HU, 2007).

Com relação à exposição ao $\mathrm{Pb}$ em crianças e aos seus valores indicativos de risco, segundo De Capitani e Paoliello (2012), apesar de não se dispor de valores de referência atualizados para fins de comparação dos resultados de estudos epidemiológicos, os trabalhos de avaliação da exposição realizados no Brasil têm utilizado os parâmetros estabelecidos pelo Centro de Controle e Prevenção de Doenças (Center of Disease Control and Prevention - $\mathrm{CDC}$ ), dos Estados Unidos, como referência para tomadas de decisão quanto a medidas de intervenção. Nesse sentido, o CDC desde 1990 estabeleceu o valor de plumbemia de $10 \mu \mathrm{g} \cdot \mathrm{dL}^{-1}$ como limite da ação para crianças. Esse valor tinha por base estudos feitos até aquele período mostrando baixo risco de danos neurológicos no longo prazo em crianças com exposições ambientais que mantivessem níveis de plumbemia abaixo desse valor (DE CAPITANI; PAOLIELLO, 2012). 
Convém ressaltar, no entanto, que no início de 2012 - Comitê Assessor para Prevenção de Intoxicação por Chumbo em Crianças (Advisory Committee on Childhood Lead Poisoning Prevention - ACCLPP) do CDC recomendou o uso do valor de referência (VR), em vez de nível de ação, como balizador das medidas de prevenção nos Estados Unidos. Nesse sentido, o CDC propõe que o nível de ação de $10,0 \mu \mathrm{g} \cdot \mathrm{dL}^{-1}$ de $\mathrm{Pb}-\mathrm{S}$ seja substituído pelo VR de $5,0 \mu \mathrm{g} . \mathrm{dL}^{-1}$, valor este derivado do percentil $97,5 \%$ dos valores obtidos em crianças de 1 a 5 anos de idade amostradas pela National Health and Nutrition Examination Survey (NHANES) (CDC, 2012). O Programa Nacional de Toxicologia (National Toxicology Program) (NTP, 2012) dos Estados Unidos afirma que existe suficiente evidência científica de que níveis de $\mathrm{Pb}$-S menores que $10,0 \mu \mathrm{g} \cdot \mathrm{dL}^{-1}$ estão associados a efeitos adversos à saúde humana, tais como déficits neurocomportamentais, diminuição do desempenho escolar e quociente de inteligência (QI).

\section{METODOLOGIA}

\section{Cenário de exposição}

O cenário de exposição definiu-se espacialmente como o conjunto de domicílios localizados próximos a uma antiga fundição primária de $\mathrm{Pb}$ inativa há mais de duas décadas, local comprovadamente contaminado por metais e caracterizado pelo histórico de utilização da escória como aterro em quintais e base de pavimentação (MACHADO et al., 2004; MACHADO et al., 2013). Trinta e oito crianças entre 0 e 7 anos de idade residentes até uma distância de 1,2 km da antiga fábrica foram selecionadas como parte do cenário de exposição. Considerando o quadro de exposição, dois cenários de avaliação foram definidos:

- cenário padronizado ou estandardizado;

- cenário individualizado.

No cenário padronizado, assume-se que a população de crianças está exposta às mesmas fontes e aos mesmos níveis ambientais de $\mathrm{Pb}$ (valores médios). O resultado da estimação, utilizando esse cenário, corresponde ao valor de $\mathrm{Pb}-\mathrm{S}$ esperado para uma criança vista como padrão sob as condições de exposição. Já o cenário individualizado permite a predição dos valores de $\mathrm{Pb}-\mathrm{S}$ individuais para cada criança, sempre e quando conhecidos os níveis ambientais de $\mathrm{Pb}$ detectados nas fontes de exposição (caso a caso), além de características próprias, tais como idade e peso corporal.

Neste estudo a população de crianças, na avaliação de ambos os cenários, foi dividida em dois grupos:

- crianças que declararam o consumo de alimentos vegetais cultivados nos seus próprios quintais;

- crianças que declararam não ter esse hábito.
Levaram-se em conta exclusivamente como fontes de exposição ao $\mathrm{Pb}$ o solo superficial de quintais, a poeira domiciliar e os alimentos vegetais cultivados em quintais da área e consumidos pela população quando declarado. Assume-se que essas variáveis estão intrinsecamente relacionadas ao quadro de contaminação remanescente do local apresentado em Rabelo (2010), Machado et al. (2013) e Magna et al. (2013).

No grupo de crianças que declararam o consumo de alimentos vegetais, foi realizada apenas a análise para o cenário padronizado, a forma de representar tendência central do risco quando confirmada essa condição.

É importante destacar ainda que tanto os valores de concentração de $\mathrm{Pb}$ nos compartimentos ambientais quanto os valores de $\mathrm{Pb}-\mathrm{S}$ obtidos experimentalmente foram organizados e adaptados ao presente estudo, mediante resultados alcançados entre os anos de 2009 a 2011, gerando uma base de dados que tornou possível a presente pesquisa. No Quadro 1, estão os trabaIhos utilizados na produção da base de dados.

A obtenção de todas as informações relacionadas com as crianças participantes seguiu os princípios éticos estabelecidos pela Resolução no 196/96 do Ministério da Saúde, que trata do gerenciamento de pesquisas que envolvem seres humanos, tendo a devida aprovação dos comitês de Ética em Pesquisa do Centro de Pesquisa Gonçalo Muniz, da Fundação Oswaldo Cruz (protocolo no 324, parecer no 217/2010), e da Universidade Católica do Salvador (ofício no 0073/10).

Na Tabela 1, apresenta-se um resumo das concentrações de $\mathrm{Pb}$ detectadas nos compartimentos ambientais, caracterizando em parte o cenário de exposição. 


\section{Calibração dos modelos}

$\mathrm{Na}$ estimativa dos valores de $\mathrm{Pb}-\mathrm{S}$ por meio dos modelos sob avaliação, foi utilizada uma sequência consecutiva de etapas, iniciando-se a simulação mediante o emprego do cenário padronizado (valores médios de $\mathrm{Pb}$ detectados nos compartimentos ambientais) e os parâmetros predefinidos pelo modelo IEUBK. Nessa avaliação inicial, foram apenas consideradas as rotas de exposição solo e poeira domiciliar, excluindo-se a ingestão de alimentos vegetais, já que a princípio se tinha como objetivo definir os parâmetros de taxas de ingestão (solo e poeira) pelas crianças e a biodisponibilidade do contaminante nessas rotas. Vale ressaltar que no presente estudo os valores de parâmetros de entrada foram obtidos, sempre que possível, de dados experimentais adquiridos de estudos realizados na área. No caso da não existência de valores experimentais, dados de literatura foram utilizados. Na Tabela 2, são apresentados os valores definitivos usados na avaliação.

O processo de calibração iniciou-se mediante o uso do modelo IEUBK e de seus parâmetros de entrada predefinidos complementares (Tabela 2), além dos valores médios de $\mathrm{Pb}$ detectados nas rotas de exposição avaliadas. Foram utilizados como indicadores-guia o valor médio de $\mathrm{Pb}-\mathrm{S}$ determinado experimentalmente e o percentual de valores detectados acima de $5,0 \mu \mathrm{g} . \mathrm{dL}^{-1}$, valores que foram comparados com os estimados pelo IEUBK. Após as análises preliminares, os valores de biodisponibilidade foram sendo reduzidos em passos de $10 \%$, de forma iterativa, até alcançarem um valor de $\mathrm{Pb}-\mathrm{S}$ próximo ao determinado experimentalmente. A redução dos valores predefinidos de biodisponibilidade, no presente estudo, esteve sustentada em antecedentes referidos às características do solo local, aos ensaios de coluna desenvolvidos no Projeto Purifica (MACHADO, 2003), que demostraram a elevada capacidade de atenuação natural do solo (valor de coeficiente de retardo $R=52,7$ ), e aos baixos fatores de transferência solo-planta para $\mathrm{Pb}$ determinados nos alimentos vegetais da área de estudo (MAGNA et al., 2013). Na Figura 1, apresenta-se o fluxograma do processo de calibração.

Com o intuito de possibilitar a comparação entre os resultados produzidos pelos modelos e a utilização das concentrações de $\mathrm{Pb}-\mathrm{S}$ como biomarcador de ex-

\section{Quadro 1 - Estudos utilizados na produção da base de dados}

\begin{tabular}{|l|c|c|}
\hline Material avaliado & Período & Referência \\
\hline Solo superficial & 2009 a 2011 & Rabelo (2010) e Machado et al. (2013) \\
\hline Alimentos vegetais & 2009 a 2011 & Magna (2011) \\
\hline Poeira domiciliar & 2010 & Guerra (2010) \\
\hline Sangue & 2010 & Presente estudo \\
\hline
\end{tabular}

Tabela 1 - Concentrações de chumbo detectadas nos compartimentos ambientais no local de estudo.

\begin{tabular}{|l|c|c|c|}
\hline & $\begin{array}{c}\text { Solo } \\
\left(\mathbf{m g} \cdot \mathbf{k g}^{-1}\right)\end{array}$ & $\begin{array}{c}\text { Poeira domiciliar } \\
\left(\mathbf{m g}^{-1}\right)\end{array}$ & $\begin{array}{c}\text { Alimentos vegetais } \\
\left(\mathbf{m g}^{-1} \mathbf{k g}^{-1}\right)\end{array}$ \\
\hline Mínimo & 60,00 & 71,60 & 0,18 \\
\hline Média & $1.096,00$ & 219,42 & 19,60 \\
\hline Desvio padrão & 919,90 & 105,35 & 6,20 \\
\hline Máximo & $2.971,00$ & 499,16 & 118,20 \\
\hline $\mathrm{n}$ & 39 & 38 & 57 \\
\hline
\end{tabular}

Fonte: Rabelo (2010), Guerra (2010), Magna (2011). 
posição, foi necessário adicionar uma nova etapa ao processo de avaliação do risco no modelo tradicional da ATSDR, que não fornece diretamente valores de $\mathrm{Pb}-\mathrm{S}$. Dessa forma, de posse dos resultados da ATSDR, empregou-se a relação dose de referência e concentração de $\mathrm{Pb}-\mathrm{S}$ reportada pela World Health Organization (WHO, 1993) (25 $\mu \mathrm{g} \cdot \mathrm{kg}^{-1} \mathrm{semana}^{-1}$ corresponde a $5,70 \mu \mathrm{g} \cdot \mathrm{dL}^{-1}$ ) para estimar os valores de $\mathrm{Pb}-\mathrm{S}$ previstos.

Tabela 2 - Parâmetros de entrada utilizados.

\begin{tabular}{|l|c|c|}
\hline Parâmetro & Valor & Observação \\
\hline Concentração de chumbo no $\mathrm{ar}\left(\mu \mathrm{g} / \mathrm{m}^{3}\right)$ & 0,1 & Padrão \\
\hline Consumo de água (L/dia)* & $0,20-0,59$ & Padrão \\
\hline Ingestão de solo e poeira $(\mathrm{g} / \mathrm{dia})^{*}$ & $0,085-0,135$ & Padrão \\
\hline Ingestão de chumbo na dieta $(\mu \mathrm{g} / \mathrm{dia})^{*}$ & $2,04-2,26$ & Padrão \\
\hline Horas no ambiente exterior $(\mathrm{hr} / \mathrm{dia})^{* *}$ & 6 & Modificado \\
\hline Porcentagem solo/poeira $(\%)$ & 40 & Modificado \\
\hline Porcentagem de vegetais provenientes do quintal $(\%)^{* * *}$ & 14 & Modificado \\
\hline
\end{tabular}

Padrão: valor predefinido no modelo integrated exposure uptake biokinetic (IEUBK); Modificado: alterado em função dos testes de verificação realizados; *valores apresentam variações em função das faixas etárias; **valor utilizado para crianças maiores de 4 anos de idade em função das condições climáticas do local; ***valor baseado no estudo desenvolvido por Garrote (2004).

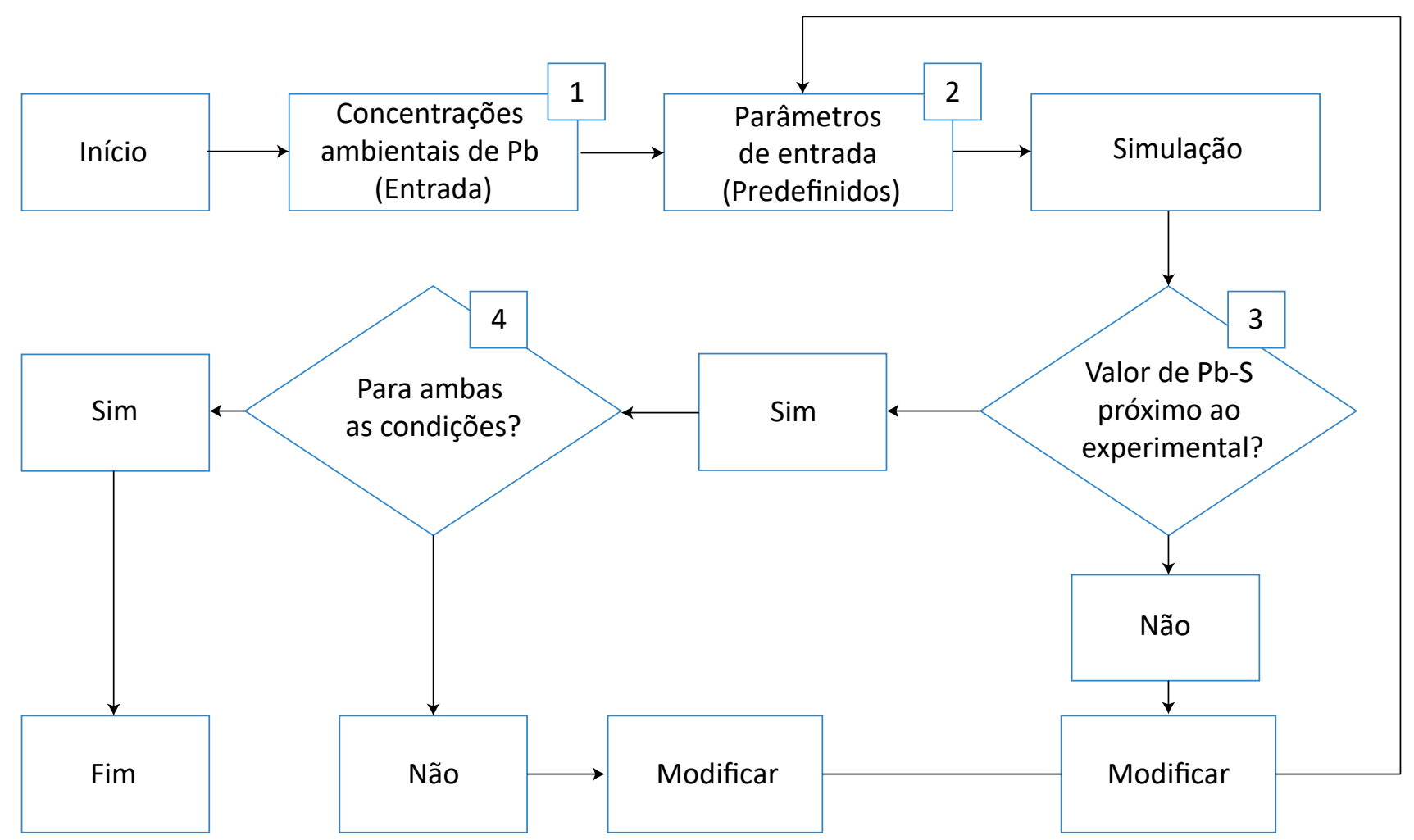

$\mathrm{Pb}$ : chumbo; ${ }^{1}$ valores correspondentes ao cenário padrão; ${ }^{2}$ valores apresentados na Tabela $2 ;{ }^{3}$ valor da média geométrica de $\mathrm{Pb}$ no sangue $(\mathrm{Pb}-\mathrm{S})$ determinado de forma experimental; ${ }^{4}$ condição referente ao consumo ou não de alimentos vegetais.

Figura 1 - Processo de calibração de modelos de riscos. 
Vale ressaltar que o valor de dose estimada usada para a transformação correspondeu à dose média total de exposição ao $\mathrm{Pb}$ pela via de ingestão, calculada por meio da Equação 1.

$D_{\text {exp-total }}=D_{\text {exp -solo }}+D_{\text {exp-poeira }}+D_{\exp -\text { alim }}+D_{\text {exp-dieta }}$

Em que:

$\mathrm{D}_{\text {exp-total }}=$ a dose de exposição total ao contaminante de interesse $\left(\mathrm{mg} \mathrm{kg}^{-1} \mathrm{dia}^{-1}\right)$;

$D_{\text {exp-solo }}=$ a dose de exposição ao solo contaminado $\left(\mathrm{mg}^{\mathrm{kg}}{ }^{-1} \mathrm{dia}^{-1}\right)$;

\section{Análise da capacidade preditiva}

A análise da capacidade preditiva dos valores de $\mathrm{Pb}-\mathrm{S}$ foi realizada de forma sequencial em duas fases. A primeira iniciou-se com a avaliação da capacidade de predição individual de cada modelo para ambos os cenários, quando possível, observando-se sempre o critério de consumo de vegetais e comparando-se os

\section{Tratamento estatístico de dados}

Medidas de tendência central, tais como média aritmética (média) e geométrica, foram utilizadas no cenário padronizado de avaliação. Já no cenário individualizado, além das medidas de tendência central mencionadas anteriormente, estimaram-se medidas de dispersão, como valores mínimos e máximos e des-
$\mathrm{D}_{\text {exp-poeira }}=\mathrm{a}$ dose de exposição à poeira contaminada (mg. kg $\left.{ }^{-1} \mathrm{dia}^{-1}\right)$;

$\mathrm{D}_{\text {exp-alim }}=$ a dose de exposição pela ingestão de alimentos vegetais contaminados (mg. $\left.\mathrm{kg}^{-1} \mathrm{dia}^{-1}\right)$;

$D_{\text {exp-dieta }}=$ a dose de exposição ao contaminante pela ingestão na dieta $\left(\mathrm{mg} \cdot \mathrm{kg}^{-1} \mathrm{dia}^{-1}\right)$.

Obtidos os valores de $\mathrm{Pb}-\mathrm{S}$ previstos, o modelo da ATSDR foi finalmente ajustado de forma análoga ao IEUBK. Calibrados ambos os modelos, foram estimadas as concentrações de $\mathrm{Pb}$-S. Na Tabela 3 são resumidos os valores de biodisponibilidade definitivos obtidos no processo de calibração segundo rota de exposição e modelo.

valores previstos com os de $\mathrm{Pb}-\mathrm{S}$ determinados experimentalmente. Já na segunda fase, continuou-se com a comparação da capacidade preditiva entre modelos considerando apenas o cenário individualizado para o grupo de crianças que negou o consumo de alimentos vegetais (ATSDR comparado com IEUBK).

vio padrão, para a comparação dos resultados de $\mathrm{Pb}-\mathrm{S}$. Com o objetivo de observar diferenças estatisticamente significativas nos valores médios de $\mathrm{Pb}-\mathrm{S}$ estimados por ambos os modelos, foram aplicados os testes não paramétricos para comparação de médias U de Mann-Whitney e de Kruskal-Wallis.

Tabela 3 - Valores de biodisponibilidade de chumbo (Pb) dos modelos da Agência de Substâncias Tóxicas e Registro de Doenças (ATSDR) e modelo biocinético e de exposição integrado (IEUBK).

\begin{tabular}{|l|c|c|c|}
\hline \multirow{2}{*}{ Rota de exposição } & \multicolumn{2}{|c|}{ Biodisponibilidade (\%) } \\
\cline { 2 - 4 } & ATSDR & IEUBK & $* *$ \\
\hline Alimentos vegetais & 30 & 20 \\
\hline Solo superficial & 20 & 20 \\
\hline Poeira domiciliar & 20 & 10 \\
\hline Dieta alimentar & $*$ & 30 \\
\hline *O modelo da ATSDR não permite o cálculo direto de doses de exposição via dieta alimentar; **no modelo IEUBK a biodisponibilidade do Pb presen- \\
te nos alimentos vegetais é considerada como parte da biodisponibilidade do metal na dieta alimentar; ***valor predefinido pelo software IEUBK.
\end{tabular}


Na comparação dos resultados obtidos entre modelos, foram realizadas análises de correlação linear simples, expondo as associações observadas mediante diagramas de dispersão e quantificadas por meio dos coeficientes de correlação de Pearson ( $r$ ) e do seu $p(\alpha)$, além do coeficiente de determinação $\left(r^{2}\right)$. Os intervalos de valores do coeficiente de correlação de Pearson ( $r$ ) empregados neste estudo são apresentados na Tabela 4 e decorrem das sugestões de interpretação de Cohen (1988) e expostas no trabalho de Lalinde et al. (2018).

\section{RESULTADOS}

\section{Níveis de $\mathrm{Pb}$-S em crianças de 0 a 7 anos}

Os resultados obtidos na determinação das concentrações de $\mathrm{Pb}$-S em crianças que declararam não consumir vegetais cultivados nos seus quintais ( $n=20$ ) ficaram na faixa de 2,28 a $7,58 \mu \mathrm{g} \cdot \mathrm{dL}^{-1}$, com valor médio de 4,55 $\mu \mathrm{g} \cdot \mathrm{dL}^{-1}$, desvio padrão de 1,42 $\mu \mathrm{g} \cdot \mathrm{dL}^{-1}$ e média geométrica de $4,34 \mu \mathrm{g} \cdot \mathrm{dL}^{-1}$. Aproximadamente $35 \%$ dos valores obtidos se apresentaram superiores a $5 \mu \mathrm{g} \cdot \mathrm{dL}^{-1}$, valor recomendado pelo CDC (2012).

No segundo grupo de crianças, que declarou consumir alimentos vegetais provenientes de quintais $(n=18)$, os valores de $\mathrm{Pb}-\mathrm{S}$ foram detectados na faixa de 3,30 a 11,42

\section{Capacidade preditiva anterior à calibração}

\section{Cenário padronizado}

Concentrações médias de Pb-S foram obtidas mediante a utilização dos valores de entrada considerados predefinidos. Estes, em ambos os modelos, apresentaram maior aproximação às concentrações de $\mathrm{Pb}-\mathrm{S}$ no grupo de crianças $\mu \mathrm{g} \cdot \mathrm{dL}^{-1}$, com valor médio de $5,98 \mu \mathrm{g} \cdot \mathrm{dL}^{-1}$, desvio padrão de $2,84 \mu \mathrm{g} \cdot \mathrm{dL}^{-1} \mathrm{e}$ média geométrica de $5,45 \mu \mathrm{g} \cdot \mathrm{dL}^{-1}$. Das concentrações, $52,60 \%$ foram detectadas acima de $5 \mu \mathrm{g} \cdot \mathrm{dL}^{-1}$.

Em ambos os grupos avaliados, é possível observar um percentual considerável de crianças com concentrações de $\mathrm{Pb}-\mathrm{S}$ acima do valor recomendado pelo CDC (2012), vinculado a efeitos sobre o QI em crianças, sendo sempre maior esse valor quando declarado o consumo de alimentos vegetais produzidos em quintais, refletindo a possível influência desse hábito nos valores detectados.

Tabela 4 - Interpretação do coeficiente de correlação de Pearson (r).

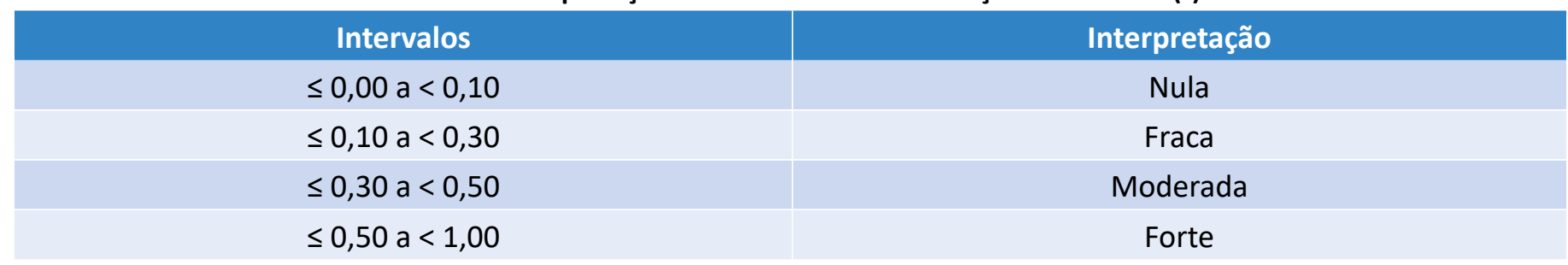

Tabela 5 - Valores médios de chumbo no sangue (Pb-S) estimados anteriormente à calibração dos modelos: cenário padronizado.

\begin{tabular}{|c|c|c|c|}
\hline \multirow{2}{*}{ Condição } & \multirow{2}{*}{ Modelos } & \multicolumn{2}{|c|}{$\mathrm{Pb}-\mathrm{S}\left(\mu \mathrm{g} \cdot \mathrm{dL}^{-1}\right.$ ) } \\
\hline & & Previsto & Experimental \\
\hline \multirow{2}{*}{ Sem consumo de alimentos vegetais } & ATSDR & 3,92 & 4,56 \\
\hline & IEUBK & $6,17^{*}$ & $4,34 *$ \\
\hline \multirow{2}{*}{ Com consumo de alimentos vegetais } & ATSDR & 8,71 & 5,98 \\
\hline & IEUBK & $21,4^{*}$ & $5,45^{*}$ \\
\hline
\end{tabular}

*média geométrica de Pb-S; ATSDR: Agência de Substâncias Tóxicas e Registro de Doenças; IEUBK: integrated exposure uptake biokinetic. 
Da mesma forma, o resultado do percentual de crianças com concentrações de $\mathrm{Pb}-\mathrm{S}$ acima de $5 \mu \mathrm{g} \cdot \mathrm{dL}^{-1}$, estimado pelo IEUBK anterior à calibração, foi de $67,30 \%$, sendo possível observar a diferença quando comparado com o percentual obtido nos valores experimentais de $35 \%$. De acordo com Brattin e Griffin (2011), o uso de valores predefinidos pelo IEUBK é comumente considerado conservador, já que a sua

\section{Cenário individualizado}

No cenário individualizado, e considerando apenas as crianças que declararam não consumir alimentos vegetais, é possível verificar diferenças nos valores para ambos os modelos quando comparados com os valores obtidos experimentalmente (Tabela 6). utilização provavelmente resulta em superestimação da exposição infantil ao $\mathrm{Pb}$ pelo solo contaminado. Nesse sentido, Romieu et al. (1997) explicam que a aplicação do modelo IEUBK em locais contaminados na América Latina apresenta alto potencial para prever níveis de $\mathrm{Pb}$-S em crianças sempre e quando dados do cenário de contaminação são corretamente determinados e utilizados.

A análise apresentada aponta a importância e a necessidade de realizar uma calibração adequada dos modelos, de maneira a não superestimar as observações e melhorar a exatidão da capacidade preditiva.

\section{Capacidade preditiva individual: modelo da ATSDR}

\section{Cenário padronizado}

Utilizando a relação entre o valor da dose de referência para $\mathrm{Pb}$ e a concentração de $\mathrm{Pb}-\mathrm{S}$ exposta pela WHO (1993) e a dose total média de exposição estimada para crianças de 0 a 7 anos de idade que consomem alimentos vegetais, chega-se ao valor previsto de $7,66 \mu \mathrm{g} \cdot \mathrm{dL}^{-1}$, superior à concentração média detectada experimental-

\section{Cenário individualizado}

Avaliando os valores individuais de $\mathrm{Pb}-\mathrm{S}$ relativos a crianças que declararam não consumir alimentos vegetais produzidos em quintais, o valor médio de $\mathrm{Pb}-\mathrm{S}$ estimado foi de $4,65 \mu \mathrm{g} \cdot \mathrm{dL}^{-1}$, próximo ao detectado experimentalmente, de 4,55 $\mu \mathrm{g} \cdot \mathrm{dL}^{-1}$. Aplicando o teste de Mann-Whitney, não foram detectadas diferenças estatisticamente significativas nos valores médios de $\mathrm{Pb}-\mathrm{S}$ quando comparados com os valores determinados experimentalmente $(p=0,927)$. Na Tabela 7 se apresentam os resultados obtidos nessa análise. mente nesse grupo de crianças $\left(5,98 \mu \mathrm{g} \cdot \mathrm{dL}^{-1}\right)$. Comparando a concentração média de $\mathrm{Pb}-\mathrm{S}$ em crianças que não possuem o hábito de consumir alimentos vegetais produzidos em quintais, o valor estimado foi de $4,25 \mu \mathrm{g} \cdot \mathrm{dL}^{-1}$, aproximando-se do valor médio detectado experimentalmente, de 4,55 $\mu \mathrm{g} . \mathrm{dL}^{-1}$.

Quando comparados graficamente os valores determinados experimentalmente e os estimados pelo modelo da ATSDR (derivados das doses individuais), foi observada relação linear moderada entre ambos os valores $\left(r=0,47 ; r^{2}=0,22\right)$. Na Figura 2 se vê essa associação.

Aparentemente as estimações realizadas, considerando o cenário individualizado, refletem melhor a contribuição das rotas de exposição avaliadas para as concentrações de $\mathrm{Pb}-\mathrm{S}$.

Tabela 6 - Valores médios de chumbo no sangue (Pb-S) estimados anteriormente à calibração dos modelos: cenário individualizado.

\begin{tabular}{|c|c|c|c|c|c|c|}
\hline \multirow{2}{*}{ Condição } & \multirow{2}{*}{ Modelos } & \multicolumn{5}{|c|}{ Pb-S ( $\left.\mu g \cdot d^{-1}\right)$} \\
\hline & & Mín & Máx & Média (DP) & MG & $\mathbf{n}$ \\
\hline \multirow{3}{*}{ Sem consumo de alimentos vegetais } & ATSDR & 1,70 & 7,27 & $3,92(1,81)$ & 3,55 & 17 \\
\hline & IEUBK & 1,30 & 15,21 & $6,48(3,95)$ & 5,34 & 20 \\
\hline & Experimental & 2,28 & 7,50 & $4,55(1,42)$ & 4,34 & 20 \\
\hline
\end{tabular}

Mín: mínimo; Máx: máximo; DP: desvio padrão; MG: média geométrica; ATSDR: Agência de Substâncias Tóxicas e Registro de Doenças; IEUBK: integrated exposure uptake biokinetic. 


\section{Capacidade preditiva individual: modelo IEUBK}

\section{Cenário padronizado}

$\mathrm{O}$ valor da média geométrica de $\mathrm{Pb}-\mathrm{S}$ estimada pelo modelo IEUBK no grupo de crianças sem consumo de vegetais foi próximo ao detectado experimentalmente $\left(4,17\right.$ e $4,34 \mu \mathrm{g} \cdot \mathrm{dL}^{-1}$, respectivamente). Já a média geométrica de $\mathrm{Pb}-\mathrm{S}$ estimada para crianças que de- clararam o consumo de alimentos vegetais foi de $8,73 \mu \mathrm{g} \cdot \mathrm{dL}^{-1}$. O valor estimado é 1,6 vez a média geométrica de $\mathrm{Pb}-\mathrm{S}$ determinada nas crianças desse grupo na área de estudo $\left(5,45 \mu \mathrm{g} \cdot \mathrm{dL}^{-1}\right)$. Essa observação confirma o consumo de alimentos vegetais como uma

Tabela 7 - Valores de chumbo no sangue ( $\mathrm{Pb}-\mathrm{S})$ estimados por meio dos resultados do modelo da Agência de Substâncias Tóxicas e Registro de Doenças (ATSDR) e da determinação experimental.

\begin{tabular}{|c|c|c|c|c|c|c|c|}
\hline \multirow{2}{*}{ Condição } & \multirow{2}{*}{ Modelo } & \multicolumn{5}{|c|}{$\mathrm{Pb}-\mathrm{S}\left(\mu \mathrm{g} \cdot \mathrm{dL}^{-1}\right)$} & \multirow{2}{*}{$\begin{array}{c}\text { Teste Mann-Whitney } \\
\text { p (sig.) }\end{array}$} \\
\hline & & Mín & Máx & Média (DP) & MG & $n$ & \\
\hline \multirow{2}{*}{$\begin{array}{l}\text { Sem consumo de } \\
\text { alimentos vegetais }\end{array}$} & ATSDR & 2,64 & 7,87 & $4,65(1,63)$ & 4,39 & 17 & \multirow{2}{*}{0,927} \\
\hline & Experimental & 2,28 & 7,50 & $4,55(1,42)$ & 4,34 & 20 & \\
\hline
\end{tabular}

Mín: mínimo; Máx: máximo; DP: desvio padrão; MG: média geométrica; $\mathrm{p}$ (sig.): significância estatística relacionada ao teste de Mann-Whitney de diferença de médias. Valores de $n$ apresentam variação em função da disponibilidade de dados para análise.

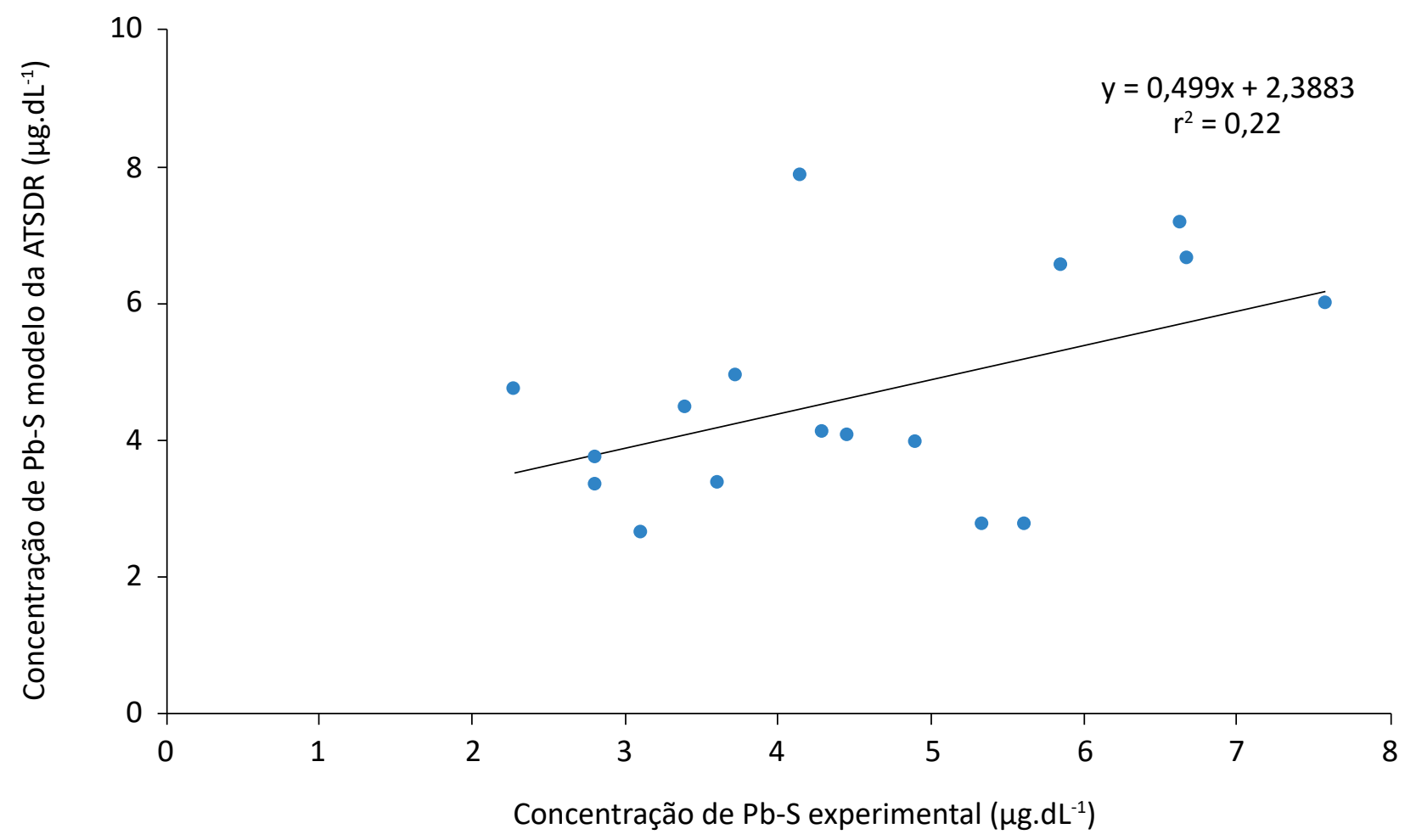

Figura 2 - Teor de chumbo no sangue (Pb-S) estimado pelo modelo da Agência de Substâncias Tóxicas e Registro de Doenças (ATSDR) comparado com o teor de Pb-S determinado experimentalmente. 
condição que influencia a estimação da concentração média de $\mathrm{Pb}$-S próxima à determinada experimentalmente nesse grupo. É provável que a espécie vegetal,

\section{Cenário individualizado}

$\mathrm{O}$ valor estimado da média geométrica de $\mathrm{Pb}-\mathrm{S}$ para o primeiro grupo (sem consumo de alimentos vegetais) foi de 3,54 $\mu \mathrm{g} \cdot \mathrm{dL}^{-1}$, sendo inferior em $22 \%$ ao valor detectado experimentalmente. Aplicando o teste de Mann-Whitney, não foram detectadas diferenças estatisticamente significativas nos valores médios de $\mathrm{Pb}-\mathrm{S}$ quando comparados à a preferência e a frequência de consumo dos alimentos vegetais determinem parte da diferença observada na estimação.

estimativa realizada mediante o IEUBK e aos valores determinados experimentalmente $(p=0,402)$. Na Tabela 8 se apresentam os resultados obtidos nessa análise.

A Figura 3 compara os teores de $\mathrm{Pb}-\mathrm{S}$ determinados nas crianças que não consomem alimentos vegetais

Tabela 8 - Comparação entre os valores de concentração de chumbo no sangue ( $\mathrm{Pb}-\mathrm{S})$ detectados experimentalmente e os estimados pelo modelo biocinético e de exposição integrado (IEUBK).

\begin{tabular}{|c|c|c|c|c|c|c|c|}
\hline \multirow{2}{*}{ Condição } & \multirow{2}{*}{ Modelo } & \multicolumn{5}{|c|}{$\mathrm{Pb}-\mathrm{S}\left(\mu \mathrm{g} \cdot \mathrm{dL}^{-1}\right)$} & Teste Mann-Whitney \\
\hline & & Mín & Máx & Média (DP) & MG & $\mathbf{n}$ & p (sig.) \\
\hline \multirow{2}{*}{$\begin{array}{l}\text { Sem consumo de } \\
\text { alimentos vegetais }\end{array}$} & IEUBK & 0,76 & 11,17 & $4,44(2,93)$ & 3,54 & 20 & \multirow{2}{*}{0,402} \\
\hline & Experimental & 2,28 & 7,50 & $4,55(1,42)$ & 4,34 & 20 & \\
\hline
\end{tabular}

Mín: mínimo, Máx: máximo; DP: desvio padrão; MG: média geométrica; p (sig.): significância estatística relacionada ao teste de Mann-Whitney de diferença de médias.

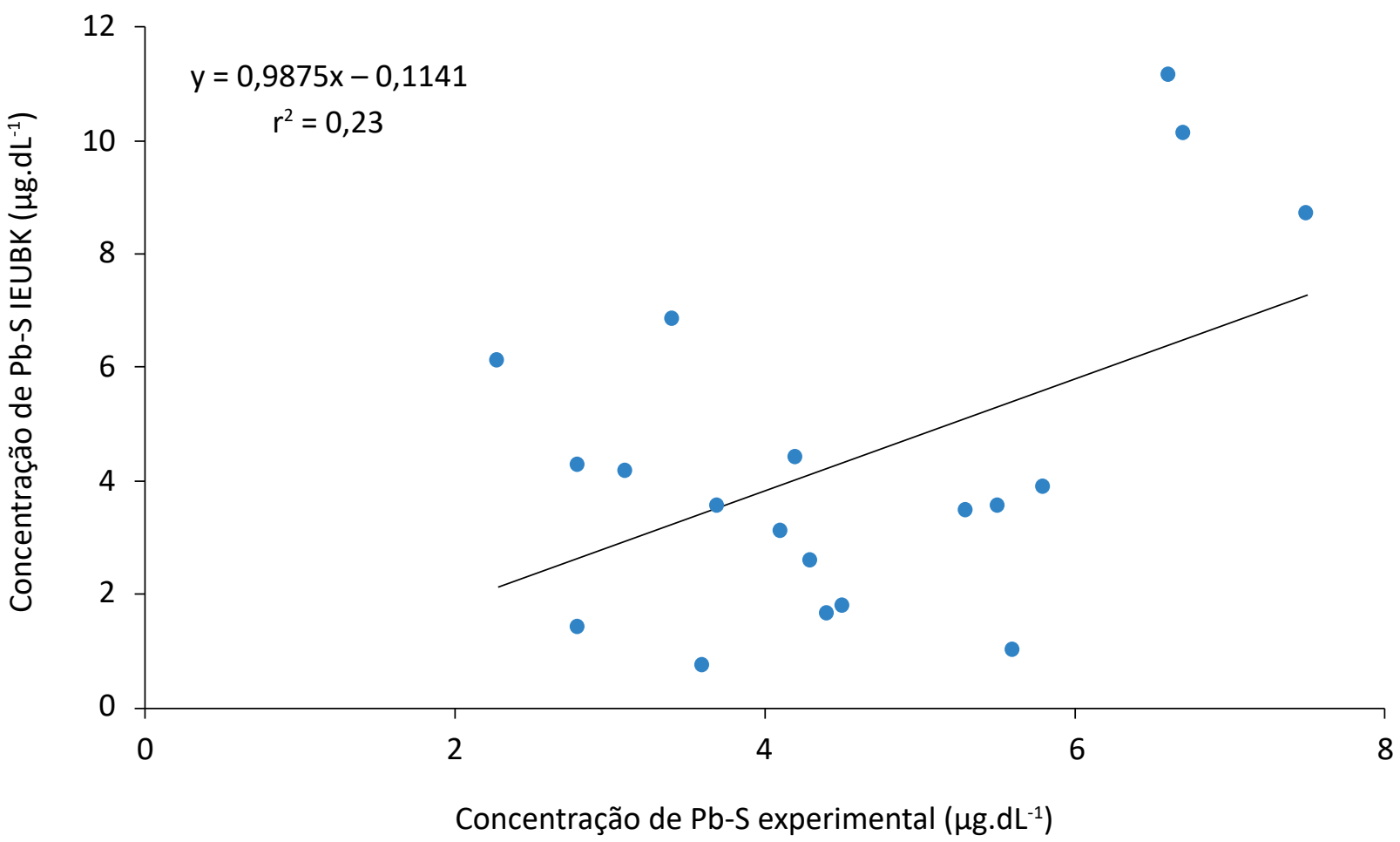

Figura 3 - Teor de chumbo no sangue (Pb-S) estimado pelo modelo biocinético e de exposição integrado (IEUBK) comparado com o teor de $\mathrm{Pb}-\mathrm{S}$ determinado experimentalmente para crianças sem consumo de alimentos vegetais. 
com os teores de $\mathrm{Pb}-\mathrm{S}$ estimados pelo IEUBK. Conforme se observa, os valores apresentados encontram-se moderadamente associados de forma linear com um coeficiente de correlação de $r=0,48\left(r^{2}=0,23\right)$.

\section{Comparação da capacidade preditiva do risco entre modelos: ATSDR versus IEUBK}

\section{Cenário individualizado}

Mediante a estimação dos valores individuais de $\mathrm{Pb}-\mathrm{S}$ e sua respectiva comparação de valores médios, não foram observadas diferenças estatisticamente significativas nos valores de $\mathrm{Pb}-\mathrm{S}$ quando estimados por ambos os modelos $(p=0,393)$. Na Tabela 9 , apresentam-se os valores obtidos segundo o modelo utilizado.

Pode-se observar na Tabela 9 que ambos os modelos preveem percentuais similares de concentrações acima de $5 \mu \mathrm{g} \cdot \mathrm{dL}^{-1}$, sendo $29 \%$ para o modelo IEUBK,
$30 \%$ para o modelo da ATSDR e 35\% para a determinação experimental.

Na Figura 4, estão os resultados obtidos quando comparados os valores de $\mathrm{Pb}-\mathrm{S}$ estimados pelo IEUBK e os obtidos mediante estimação da ATSDR para o grupo de crianças expostas ao $\mathrm{Pb}$ exclusivamente pelas rotas solo, poeira domiciliar e dieta alimentar. É possível observar associação linear forte entre os valores de $\mathrm{Pb}-\mathrm{S}$ estimados em função dos resultados da ATSDR e os valores preditos pelo modelo IEUBK $\left(r=0,60 ; r^{2}=0,38\right)$.

\section{DISCUSSÃO}

Tanto o modelo tradicional de avaliação de risco da ATSDR quanto o modelo alternativo IEUBK permitem a predição do risco quando avaliada a exposição ao $\mathrm{Pb}$. Com a utilização do modelo da ATSDR, foi possível observar a dificuldade que impõe a impossibilidade de confrontar diretamente os valores das doses de exposição estimadas e o valor de $\mathrm{Pb}-\mathrm{S}$ tido como indicador de exposição e potencial risco à saúde das crianças. Nesse contexto, foi necessário adicionar uma nova etapa ao processo de avaliação do risco da ATSDR baseada na relação dose de referência e concentração de $\mathrm{Pb}-\mathrm{S}$ reportada pela WHO (1993), condição que requer maior esforço e critério de julgamento. Essa condição relaciona-se ao tipo de dose considerada no modelo da ATSDR, o qual utiliza a dose administrada na estimação do risco, enquanto o modelo IEUBK emprega a dose absorvida.
Ressalta-se que o modelo IEUBK permite a comparação direta dos valores médios e individuais (caso a caso) de $\mathrm{Pb}-\mathrm{S}$ estimados com os valores de $\mathrm{Pb}-\mathrm{S}$ determinados experimentalmente nas crianças (biomarcador de exposição), mostrando-se mais prático na avaliação e apresentando de acordo com os resultados obtidos melhor exatidão quando comparado ao modelo da ATSDR.

Haja vista essa situação, foi de fundamental importância o biomarcador de exposição para $\mathrm{Pb}-\mathrm{S}$, determinado experimentalmente, visando à verificação das estimações. É importante destacar que a inclusão e a utilização do biomarcador de exposição ( $\mathrm{Pb}-\mathrm{S})$ como parte do processo de avaliação de riscos não constitui obrigatoriedade nos modelos sob estudo, sendo uma etapa complementar adicionada neste trabalho. Segundo Cornelis et al.

Tabela 9 - Comparação entre valores de concentração de chumbo no sangue ( $\mathrm{Pb}$-S) estimados pelo modelo da Agência de Substâncias Tóxicas e Registro de Doenças (ATSDR) e pelo modelo biocinético e de exposição integrado (IEUBK).

\begin{tabular}{|c|c|c|c|c|c|c|c|c|}
\hline Condição & Modelo & \multicolumn{6}{|c|}{$\mathrm{Pb}-\mathrm{S}(\mu \mathrm{g} / \mathrm{dL})$} & Teste Mann-Whitney \\
\hline \multirow{2}{*}{$\begin{array}{l}\text { Sem consumo de } \\
\text { alimentos vegetais }\end{array}$} & IEUBK & 0,76 & 11,17 & $4,44(2,93)$ & 3,54 & 20 & 30 & \multirow{2}{*}{0,393} \\
\hline & ATSDR & 2,64 & 7,87 & $4,65(1,63)$ & 4,39 & 17 & 29 & \\
\hline
\end{tabular}

Mín: mínimo, Máx: máximo; DP: desvio padrão; MG: média geométrica; p (sig.): significância estatística relacionada ao teste de Mann-Whitney de diferença de médias. 
(2006), os biomarcadores de exposição permitem quantificar a carga corporal de um contaminante, mas não permitem identificar as fontes de exposição, confirmando a complementaridade dos métodos, quando possíveis de serem empregados. Na Tabela 10 se apresenta um resumo das estimativas para o cenário padronizado.

Por meio da etapa de calibração de ambos os modelos, foram obtidas informações adicionais neces- sárias sobre o cenário em análise, tais como valores de taxas de contato e biodisponibilidade das rotas de exposição, condição que permite obter meIhor estimação, compreensão e aproximação do real quadro de contaminação na área. Nessa perspectiva, Thums, Farago e Thornton (2008) avaliaram o valor de biodisponibilidade de $\mathrm{Pb}$ em solo (30\%), predefinido pelo IEUBK, e seu valor modificado (35 a 39\%), obtido mediante ensaios experimentais, em 30 sítios

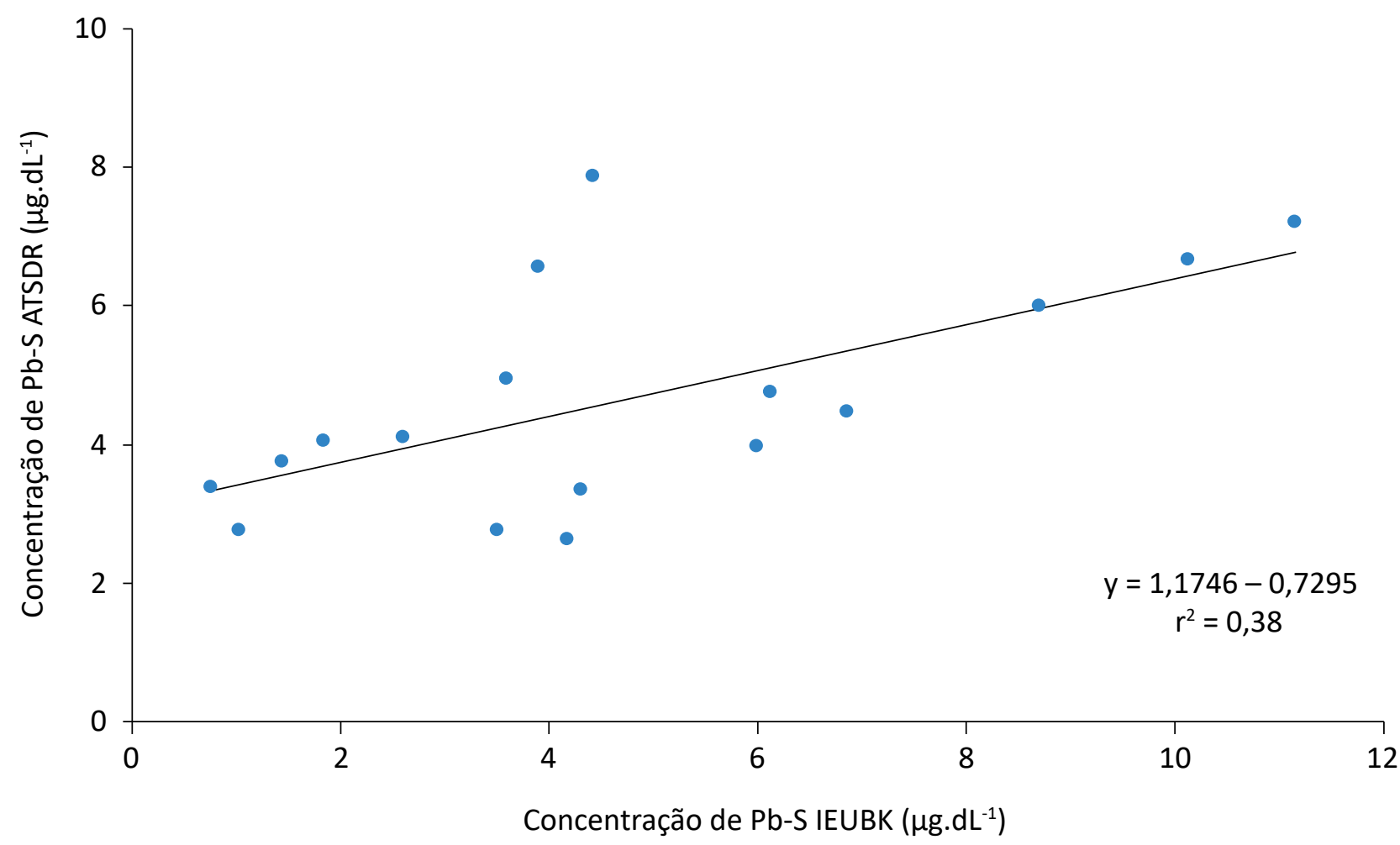

Figura 4 - Concentrações de chumbo no sangue (Pb-S) estimadas pelo modelo da Agência de Substâncias Tóxicas e Registro de Doenças (ATSDR) comparadas com as concentrações de Pb-S estimadas pelo modelo biocinético e de exposição integrado (IEUBK): grupo sem consumo de alimentos vegetais.

Tabela 10 - Comparação entre as estimativas do conteúdo médio de chumbo no sangue ( $\mathrm{Pb}-\mathrm{S})$ em crianças de 0 a 7 anos segundo modelos de risco analisados.

\begin{tabular}{|l|c|c|c|c|}
\multirow{2}{*}{ Condição } & Modelo ATSDR & Modelo IEUBK** & \multicolumn{2}{|c|}{ Pb-S experimental } \\
\cline { 2 - 5 } & Média $\left(\mu \mathrm{g} \cdot \mathrm{dL}^{-1}\right)$ & MG $\left(\mu \mathrm{g} \cdot \mathrm{dL}^{-1}\right)$ & Média $\left(\mu \mathrm{g} \cdot \mathrm{dL}^{-1}\right)$ & $\mathrm{MG}\left(\mu \mathrm{g} \cdot \mathrm{dL}^{-1}\right)$ \\
\hline Sem consumo de alimentos vegetais & 4,65 & 4,17 & 4,55 & 4,34 \\
\hline Com consumo de alimentos vegetais & 7,66 & 8,73 & 5,98 & 5,45
\end{tabular}

*Comparação em relação ao valor médio de Pb-S; **comparação em relação ao valor da média geométrica de Pb-S; ATSDR: Agência de Substâncias Tóxicas e Registro de Doenças; IEUBK: integrated exposure uptake biokinetic. 
contaminados. Na avaliação, foi constatada a ausência de valores que excederam o nível limite de $\mathrm{Pb}-\mathrm{S}$, empregado pelos autores em crianças $\left(10 \mu \mathrm{g} \cdot \mathrm{dL}^{-1}\right)$, quando usado o valor de biodisponibilidade predefinido pelo IEUBK. No entanto, utilizando os valores modificados, três locais apresentaram níveis previstos de $\mathrm{Pb}-\mathrm{S}$ excedendo o valor limite.

Situação similar foi observada por Hilts (2003), que avaliou o impacto nos níveis de Pb-S em crianças após a implementação de medidas mitigadoras relacionadas às emissões atmosféricas e posterior inativação temporária de uma fundição de $\mathrm{Pb}$ nos Estados Unidos. Os resultados apresentados pelo autor expõem que a alteração no valor do parâmetro de biodisponibilidade do solo (redução de 30 para 10\%) aproximou os níveis previstos de $\mathrm{Pb}-\mathrm{S}$ em crianças residentes na área de estudo quando comparados com níveis experimentais.

Da mesma forma, Brattin e Griffin (2011) analisaram o parâmetro predefinido pelo IEUBK relacionado à fração em massa da poeira domiciliar, que é derivada do solo exterior, em três locais contaminados por $\mathrm{Pb}$. Os autores concluíram que o valor predefinido pelo $\operatorname{IEUBK}(0,7)$ é provavelmente maior que o valor associado ao cenário de exposição e que um valor que considera as características próprias da exposição fornece maior precisão nas estimativas de risco.

Tanto os resultados obtidos no presente estudo quanto os apresentados anteriormente confirmam a necessidade da realização do processo de calibração dos modelos antes de serem utilizados na avaliação do risco.

\section{CONCLUSÕES}

A calibração dos modelos, além da utilização de valores próprios para o cenário de exposição, apresentou-se como um requisito necessário para a obtenção da previsão do risco representativo para as crianças residentes na área.

O modelo IEUBK proporcionou maior praticidade relacionada à economia de recursos e tempo para predizer o risco pela exposição ao $\mathrm{Pb}$ quando comparado ao modelo da ATSDR, contudo os dois apresentaram adequada predição do risco.
Apesar de ambos os modelos empregados permitirem a avaliação do risco pela exposição ao $\mathrm{Pb}$, a inclusão do biomarcador de exposição $(\mathrm{Pb}-\mathrm{S})$ foi de fundamental importância e permitiu reduzir consideravelmente as incertezas da análise, pelo que se recomenda o seu uso como etapa complementar nos processos de avaliação de risco à saúde pela exposição ao $\mathrm{Pb}$ em crianças de 0 a 7 anos.

Finalmente, a análise dos resultados obtidos pode fornecer um direcionamento na escolha e utilização dos modelos em problemas de contaminação de solos com potenciais efeitos sobre a saúde humana no Brasil.

\section{REFERÊNCIAS}

AGENCY FOR TOXIC SUBSTANCES AND DISEASE REGISTRY (ATSDR). Public Health Assessment: guidance manual. Atlanta: ATSDR, 2005. 357 p.

AMORIM, L.C.A. Os Biomarcadores e sua Aplicação na Avaliação da Exposição aos agentes químicos ambientais. Revista Brasileira de Epidemiologia, v. 6, n. 2, p. 1-13, 2003. http://dx.doi.org/10.1590/S1415-790X2003000200009

BARBOSA JR., F.; TANUS-SANTOS, J.E.; GERLACH, R.F.; PARSON, J.P. A Critical Review of Biomarkers Used for Monitoring Human Exposure to Lead: Advantages, Limitations, and Future Needs. Environmental Health Perspectives, v. 113, n. 12, p. 1669-1674, 2005. https://doi.org/10.1289/ehp.7917

BRATTIN, W.; GRIFFIN, S. Evaluation of the contribution of Lead in Soil to Lead in Dust at Superfund Sites. Human and Ecological Risk Assessment, v. 17, n. 1, p. 236-244, 2011. https://doi.org/10.1080/10807039.2011.538638 
CABALlERO, M.R.D.; ARAGÓN, M.C.V.; MILLAN, R.; HERRERA, M.T.A. Risk Assessment Through IEUBK Model in an Inhabited Area Contaminated with Lead. Enviromental Progress \& Sustainable Energy, v. 37, n. 1, p. 391-398, 2017. https://doi.org/10.1002/ep.12692

CAPELETI, R.A.; LEMOS, M.G.M.; DIAS, L.C.; CASARINI, C.O.D. Quantificação das Variáveis da Exposição Populacional Utilizando o Modelo de Avaliação de Risco C-SOIL para Gerenciamento de Qualidade de Solos e Águas Subterrâneas. In: CONGRESSO BRASILEIRO DE ÁGUAS SUBTERRÂNEAS, 10., 1998. Anais... São Paulo, 1998. p. 1-12.

CENTER OF DISEASE CONTROL AND PREVENTION (CDC). Low Level Lead Exposure Harms Children: A Renewed Call for Primary Prevention. Advisory Committee on Childhood Lead Poisoning Prevention of the Center of Disease Control and Prevention. Atlanta: CDC, 2012.65p.

COHEN, J. Statistical power analysis for the behavioral sciences. 2. ed. Hillsdale: Erlbaum Associates, 1988.

CORNELIS, C.; BERGHMANS, P.; SPRUNDEL, M.V.; VAN DER AUWERA, J.C. Use of the IEUBK Model for Determination of Exposure Routes in View of Site Remediation. Human and Ecological Risk Assessment, v. 12, n. 5, p. 963-982, 2006. https://doi.org/10.1080/10807030600826979

DE CAPITANI, E.M.; PAOLIELLO, B.M.M. Estudos de Avaliação da exposição ambiental humana ao chumbo no Brasil: uma análise comparativa. In: SIMPÓSIO DE ATUALIZAÇÃO CIENTIFICA DE SANTO AMARO, 1., 2012. Anais... Rio de Janeiro: CETEM, 2012. p.75-85.

DE MIGUEL, E. La Evaluación de Riesgos Ambientales. Industria y minería. Espanha: Consejo Superior de Colegios de Ingenieros de Minas de España. 2003. p. 57-64.

DÍAZ-BARRIGA, F.; ORELLANA, G. C. Metodología de Identificación y Evaluación del Riesgo para la Salud en Sitios Contaminados. Guía Metodológica. Lima, 1999. 42 p.

GARROTE, V. Os Quintais Caiçaras, Suas Características Sócio-Ambientais e Perspectivas para a Comunidade do Saco de Mamangua, Paraty, RJ. 198f. Dissertação (Mestrado) - Universidade de São Paulo, Piracicaba, 2004.

GUERRA, C. S. Utilização de dentes decíduos de regiões com diferentes históricos de contaminação ambiental para deteç̧ão de grupos de crianças expostas ao chumbo no Brasil. 201f. Tese (Doutorado) - Universidade Estadual de Campinas, Campinas, 2010.

HILTS, S. R. Effect of Smelter Emission Reductions on Children's Blood Lead Levels. The Science of the Total Environment, v. 303, n. 1-2, p. 51-58, 2003. https://doi.org/10.1016/S0048-9697(02)00357-1

LALINDE, J. D. H.; CASTRO J. F. E.; TARAZONA, M. E. P.; RODRIGUEZ, J. E.; RANGEL, J. G. C.; SIERRA, C. A. T.; TORRADO, M. K. A.; SIERRA, S. M. C.; PIRELA, V. J. B. Sobre el uso adecuado del coeficiente de correlación de Pearson: definición, propiedades y suposiciones. Archivos Venezolanos de Farmacología y Terapéutica, v. 37, n. 5, p. 587-595, 2018.

MACHADO, S. L. (coord.). Projeto PURIFICA: Proposta para remediação de áreas impactadas pela atividade extrativa de chumbo em Santo Amaro, Bahia: Relatório Síntese. Salvador: Universidade Federal da Bahia, 2003. Disponível em: <http://www.geoamb.eng.ufba.br/site/sites/default/files/arquivos/relatorios/principaisconclusoespurifica.pdf>. Acesso em: 18 jul. 2018.

MACHADO, S. L.; RABELLO, T. S.; PORTELLA, R. B.; CARVALHO, M. F.; MAGNA, G. M. M. A study of the routes of contamination by lead and cadmium in Santo Amaro, Brazil. Environmental Technology, v. 34, n. 5-8, p. 559-571, 2013. https://doi.org/10.1080/09593330.2012.692717

MACHADO, S. L.; RIBEIRO, L. D.; KIPERSTOK, A.; BOTELHO, M. A. B.; CARVALHO, M. de F. Diagnóstico da contaminação por metais pesados em Santo Amaro-BA. Revista de Engenharia Sanitária e Ambiental, v. 9, n. 2, p. 140-155, 2004. 
MAGNA, G. A. M. Análise da Exposição por Chumbo e Cádmio Presentes em Alimentos Vegetais e Gramíneas no Município de Santo Amaro - BA. Caso do Passivo Ambiental da COBRAC. 176f. Dissertação (Mestrado) - Universidade Federal da Bahia, Salvador, 2011.

MAGNA, G. A. M.; MACHAdO, S. L.; PORTELLA, R. B.; CARVALHO, M. F. Chumbo e Cádmio Detectados em Alimentos Vegetais e Gramíneas no Município de Santo Amaro-Bahia. Química Nova, v. 36, n. 7, p. 989-997, 2013. http://dx.doi. org/10.1590/S0100-40422013000700012

NATIONAL TOXICOLOGY PROGRAM (NTP). Monograph on Health Effects of Low-level Lead. Estados Unidos: United States Department of Health and Human Service, 2012.

RABELO, T. S. Estudo das Rotas Remanescentes de Contaminação por Chumbo e Cádmio no Município de Santo AmaroBA. 154f. Dissertação (Mestrado) - Universidade Federal da Bahia, Salvador, 2010.

ROMIEU, I.; LACASANA, M.; MCCONNELL, R. Lead exposure in Latin America and the Caribbean. Environment and Health Perspectives, v. 105, n. 4, p. 398-405, 1997. https://doi.org/10.2307/3433336

SCHWARTZ, B. S.; HU, H. Adult Lead Exposure: Time for Change. Environmental Health Perspectives, v. 115, n. 3, p. 451454, 2007. https://doi.org/10.1289/ehp.9782

SUÁREZ, M. L.; ARIAS, L. A.; CASTAÑEDA, R. A. J. (2007) Manual de Evaluación de Riesgo Asociado a los COP. Ministerio de Ambiente, Vivienda y Desarrollo Territorial, Republica de Colombia: Manual Metodológico. Bogotá: DDSS. 59 p.

THUMS, C. R.; FARAGO, M. E.; THORNTON, I. Bioavailability of trace metals in brownfield soils in an urban area in the UK. Environmental Geochemistry and Health, v. 30, n. 6, p. 549-563, 2008. https://doi.org/10.1007/s10653-008-9185-6

UNITED STATES ENVIRONMENTAL PROTECTION AGENCY (USEPA). (2007) User's Guide for the Integrated Exposure Uptake Biokinetic Model for Lead in Children (IEUBK) Windows. Estados Unidos: USEPA. 45p.

WORLD HEALTH ORGANIZATION (WHO). (1993) $41^{\text {th }}$ Report of the Joint FAO/WHO Expert Committee on Food Additives, Technical report series no 837. Genova: WHO, 1993. 64 p. 